\title{
A new approach for improving the silicon texturing process using gas lift effect
}

\author{
M. Amouzgar, M. Kahrizi \\ Electrical and Computer Engineering Department, Concordia University, 1515 \\ St. Catherine West, EV005.139 Montreal, Quebec, Canada H3G 2W1 \\ Email: m_amouzg@ence.concordia.ca
}

\begin{abstract}
A new cost-effective and efficient approach is proposed for texturing the crystalline silicon using the gas lift effect (GLE). The advantages of this approach over the conventional ones are that significantly lower amounts of IPA is used and much shorter etching time is required to achieve the same reflectivity. GLE is generated by taking advantage of the hydrogen bubbles evolved between the silicon wafer being etched and a glass plate, placed in parallel, creating a gap of 1-2 mm. This effect then acts as a pumping mechanism detaching more bubbles from the silicon surface, accelerating them to the top and out of the system, as quickly as they are generated. Experiments were carried out with various combinations of TMAH/IPA concentrations for two different GLE conditions to analyze and determine their influence on etching time, etching rate, surface morphology and reflectivity of the textured silicon surface. The use of this new approach in surface texturing, allowed the reduction of the required IPA by $50 \%$ and etching time by more than $60 \%$ to achieve the same reflectivity. This can ultimately lead to a significant reduction in cost by increasing the efficiency of the texturing process. A combination of 3.5\% IPA and $2 \mathrm{~mm}$ GLE resulted in a textured silicon surface having a low specular Solar Weighted Reflectivity (SWR) of $0.15 \%$.
\end{abstract}

PACS 78.40.Fy, 88.40.hm

\section{Introduction}

In the solar cell industry, the surface of the silicon is textured to decrease the reflectivity. One of the most effective and least costly techniques for silicon texturing is the anisotropic wet etching of silicon surface using strong bases such as $\mathrm{KOH}$ and TMAH [1]. In order to obtain uniform surface texturing using these etchants, one must improve the wettability of the surface and facilitate the removal of the generated hydrogen bubbles from the surface. By increasing the uniformity and coverage of the textured silicon surface, more photons are absorbed resulting in the decrease of the reflectivity. In order to achieve this, the conventional approach is to use a surface active agent such as IPA that would increase the wettability and facilitate the release of hydrogen bubbles from the silicon surface $[2,3]$. However, IPA is volatile in a heated etching bath. The boiling point of IPA is around $82^{\circ} \mathrm{C}$. Therefore, at the etching temperature of $90^{\circ} \mathrm{C}$, it must be constantly added to the solution during the etching process resulting in difficult control and higher consumption of IPA. Also, with higher IPA concentrations, the etching rate starts to decrease drastically $[4,5]$. In some experiments the IPA has been eliminated all together from the etching solution and replaced by dissolved silicon or various iononic surfactants to achieve texture uniformity $[6,12]$. Other approaches include the use of other additives such as Tertiary 
Butyl Alcohol (TBA) [7, 8] or using a metal grid to trap the hydrogen bubbles as a more controlled way to achieve the same favourable outcome [9].

The root of the problem comes from the fact that the generated hydrogen bubbles, with typical diameter range of $2-3 \mathrm{~mm}$, stick to the surface long enough to interrupt the chemical reaction between the alkaline solution and the silicon surface. This gas blanketing effect is a known occurring issue in the electrochemical reactors. Industrial electrolytic cells that produce gas at one or both electrode surfaces are usually designed to take advantage of this phenomenon to generate gas lift electrolyte circulation by creating a narrow gap between the two electrode surfaces [10]. Good electrolyte circulation provides transport of electro-active species to the electrode surface as well as taking bubbles away from the electrode surface. Internal gas lift circulation in a welldesigned electrochemical cell is much more efficient than mechanically pumped circulation [11].

In this article, the effect of gas-lift circulation on the texturing process of silicon wafers has been studied. Experiments were carried out with different combinations of TMAH/IPA concentrations with two gap sizes between the two flat surfaces facing each other creating the Gas-Lift Effect (GLE) and a reference surface where no GLE exists. More experiments were carried out to determine the effect of etching time as well as etching rate with and without the presence of GLE. Detailed analysis of the surface reflectance and surface morphology was carried out to evaluate the influence of the studied parameters.

\section{Experimental procedure}

N-type (100) orientation, single side polished, clean room processed and packaged silicon wafers with a thickness of $625+/-25 \mu \mathrm{m}$ and a resistivity of 1-10 $\Omega$-cm were purchased from Silicon Inc. and used for all experiments. All samples were cleaned using the RCA process before the experiments were carried out. Glass beaker filled with TMAH was used for etching the silicon samples. Teflon basket was used to hold the silicon samples inside the glass beaker. The beaker was placed in an oil bath to maintain temperature uniformity of the etchant solution at $90^{\circ} \mathrm{C}$. This operating temperature is below the boiling point of all the etchant solutions used containing various amount of IPA and TMAH. However, precautions were taken to assure that the concentration of all volatile components in the solution remain constant for the duration of etching. Therefore, all experiments were carried out in a closed system consisting of a beaker with a flux cap cooled through an internal circulation of 5-6 $\mathrm{C}$ running water resulting in the condensation and return of any evaporated component back to the solution. The TMAH 25 wt.\% was purchased from Sachem Inc. and diluted with DI water to reach the desired 1, 2 and 3 wt.\% concentrations used in the experiments. 99\% IPA was purchased from VWR International and was added to the etching solution to obtain weight concentrations ranging between 0 to $9 \%$. Figure 1 below shows a schematic view of the apparatus used for the anisotropic etching of silicon. 


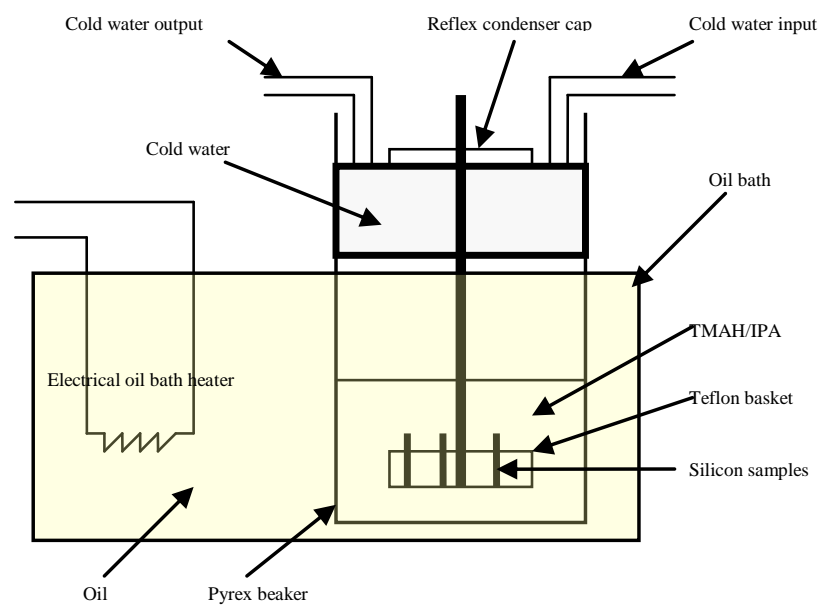

Figure 1. Schematic view of TMAH etching apparatus.

For each experiment, three Silicon wafer samples were prepared with equal dimensions of 25 (width) x 50(length) x 0.625 (thickness) $\mathrm{mm}$. Figure 2 illustrates how a piece of glass with the same length and width was used to achieve the GLE in two of the three samples with $1 \mathrm{~mm}$ and 2 $\mathrm{mm}$ gaps between the glass and the silicon wafers.

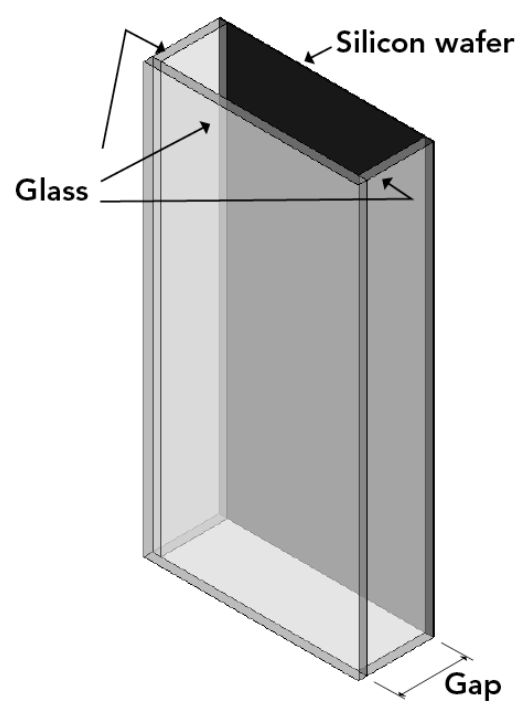

Figure 2. Schematic illustration of GLE samples used in each experiment.

Specular reflectivity of the samples was measured using a Varian Cary 5000 spectrophotometer with a baseline correction. The measured reflectivity was converted and presented here as specular Solar-Weighted Reflectance (SWR). We define the solar-weighted average reflectance as the integration of the product of the reflectance and the AM1.5 photon density, divided by the total number of photons between $300 \mathrm{~nm}$ and $1100 \mathrm{~nm}$. Etching rates were determined by weighing the samples before and after each experiment. The morphology of the textured surfaces was examined by a Hitachi S-4700 field emission scanning electron microscopy (FE-SEM). The pyramid size distribution, surface coverage and uniformity measurements were obtained by performing image analysis of the SEM photos using the ImageJ image processing program developed by the US National Institute of Health. 


\section{Results and discussion}

Anisotropic etching of silicon was performed using different TMAH (1, 2, and 3 wt.\% ) and IPA $(0,1$ and $9 \%)$ concentrations with two GLE configurations (1 and $2 \mathrm{~mm}$ gap sizes). The etchant temperature was kept constant at $90^{\circ} \mathrm{C}$ for the duration of all experiments. The GLE pumping provided agitation to help with the hydrogen bubble detachment from the surface of the silicon and to improve mass transfer of the active species to the silicon surface. The results were compared with the case where no GLE was present. The existence of GLE forced the generated hydrogen bubble to detach from the surface and leave the system as quickly as they are generated. The results in terms of surface reflectivity for three cases of no GLE, $1 \mathrm{~mm}$ and $2 \mathrm{~mm}$ gap for the previously mentioned TMAH and IPA concentrations and 30 minutes etching time are summarized in table 1.

Table 1. The effect of various IPA and TMAH concentrations on the reflectivity with and without the presence of GLE

\begin{tabular}{|l|c|c|c|c|c|c|c|c|c|}
\cline { 2 - 10 } \multicolumn{1}{c|}{} & \multicolumn{9}{c|}{ SWR (\%) } \\
\cline { 2 - 10 } \multicolumn{1}{c|}{} & \multicolumn{3}{c|}{ No GLE } & \multicolumn{3}{c|}{ 1mm GLE } & \multicolumn{3}{c|}{ 2mm GLE } \\
\cline { 2 - 10 } \multicolumn{1}{c|}{ TMAH (\%) } & \multicolumn{3}{c|}{ TMAH (\%) } & \multicolumn{3}{c|}{ TMAH (\%) } \\
\hline IPA (\%) & $\mathbf{1}$ & $\mathbf{2}$ & $\mathbf{3}$ & $\mathbf{1}$ & $\mathbf{2}$ & $\mathbf{3}$ & $\mathbf{1}$ & $\mathbf{2}$ & $\mathbf{3}$ \\
\hline $\mathbf{0}$ & 1.92 & 1.44 & 12.00 & 2.59 & 1.31 & 15.32 & 0.72 & 0.45 & 5.90 \\
\hline $\mathbf{1}$ & 0.81 & 0.62 & 7.61 & 0.96 & 0.45 & 11.39 & 0.44 & 0.39 & 1.94 \\
\hline $\mathbf{9}$ & 0.42 & 0.19 & 0.23 & 0.40 & 0.15 & 0.22 & 0.36 & 0.18 & 0.22 \\
\hline
\end{tabular}

\subsection{No GLE Present}

In order to distinguish and quantify more accurately the effect of gas lift on the texturing process, expressed or measured in terms of surface reflectivity, it was decided to first study the texturing process without the presence of GLE.

As it may be observed from table 1, as the concentration of IPA increases, a surface with a lower reflectivity is obtained for all TMAH concentrations as expected and reported in the literature [2]. We can also observe that, regardless of IPA concentration, the optimal concentration of TMAH is around $2 \%$ as previously reported [2]. However, one interesting observation from table 1 is that, the effect of IPA in improving the reflectivity is much more significant at 3\% TMAH.

It is reported in the literature that the lower texturing performance at higher concentration of TMAH may be attributed to the blocking effect of the TMA+ ions adsorbed on the surface of silicon [5]. This could also explain the higher effect of IPA at $3 \%$ TMAH compared to 2 or $1 \%$.

\subsection{Imm Gap GLE}

The same set of experiments with varying concentration of TMAH and IPA was carried out using the experimental setup, as described in section 2, to create GLE. The results of the surface reflectance for the $1 \mathrm{~mm}$ gap GLE are presented in table 1.

Consistently at 9\% IPA, the use of 1mm gap GLE improves the performance of texturing the surface resulting in better reflectivity regardless of TMAH concentration compared to the case where no GLE was used. At lower IPA concentrations (0\% and 1\%), GLE could not be sustained for the entire 30 minutes of etching time resulting in less consistent improvement in reflectivity. This could be related to the very small gap preventing the easy flow of the solution inside the gap between the Si surface and the glass not allowing the liquid to reach the expected velocity.

\subsection{2mm Gap GLE}

The results of the $2 \mathrm{~mm}$ GLE are also presented in table 1. As predicted, the increase in the gap size allowed the sustainment of the GLE throughout the 30 minutes of etching time resulting in a 
consistent improvement of texturing for all IPA concentrations. In fact, in the presence of $2 \mathrm{~mm}$ GLE, the need for the use of IPA as a surface active agent to help minimize the surface reflectivity becomes much less critical. This is also evident from the data presented in figure 3 . This figure illustrates that, the use of GLE at $0 \%$ IPA, results in $80 \%$ reduction of reflectivity compared to where there is no GLE present.

This figure illustrates that, compared with the case where there is no GLE, the $2 \mathrm{~mm}$ GLE can result in significant gains in texturing performance at low or even $0 \%$ IPA.

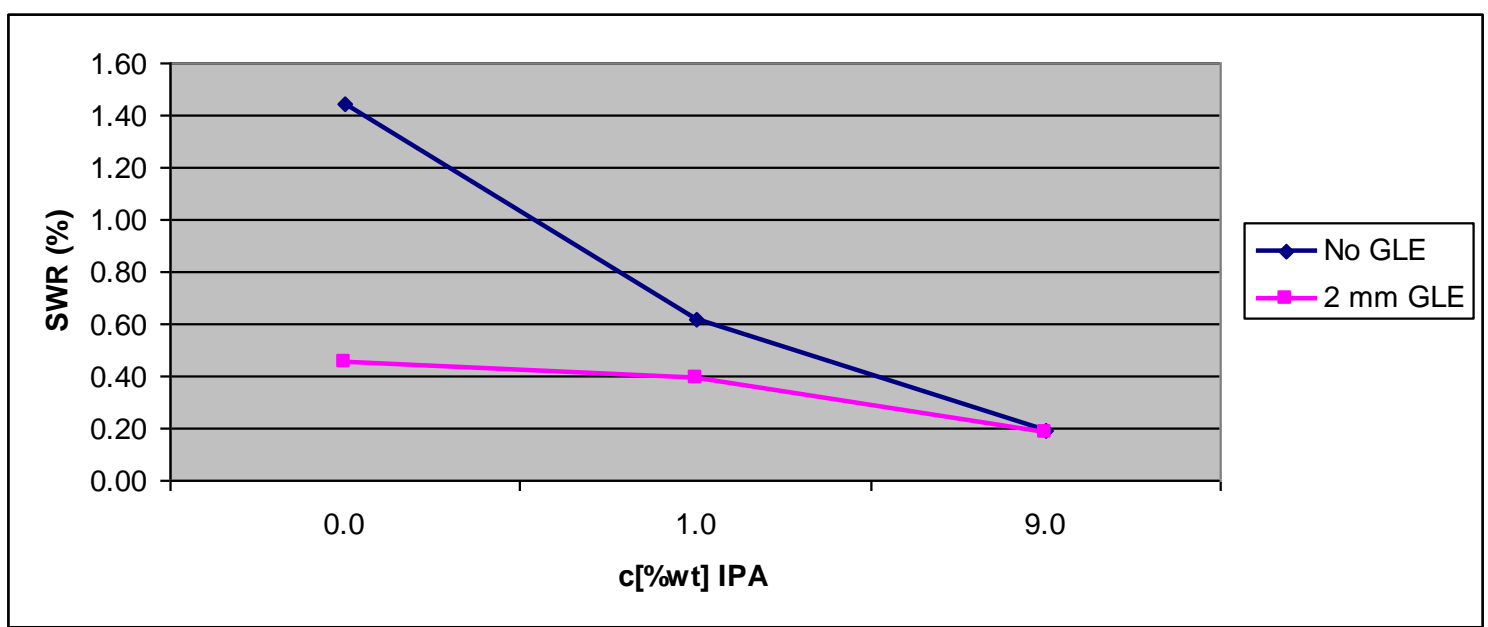

Figure 3. The use of IPA as a surface active agent becomes much less critical at the presence of the $2 \mathrm{~mm}$ GLE.

\subsection{Effect of GLE on Etching Time}

Another beneficial effect of GLE is the reduction of the etching time. In this set of experiment, the TMAH and IPA concentrations were set at $2 \%$ and $9 \%$, respectively. The temperature was kept at $90^{\circ} \mathrm{C}$ and the etching times were chosen to be 5, 10, 20 and 30 minutes.

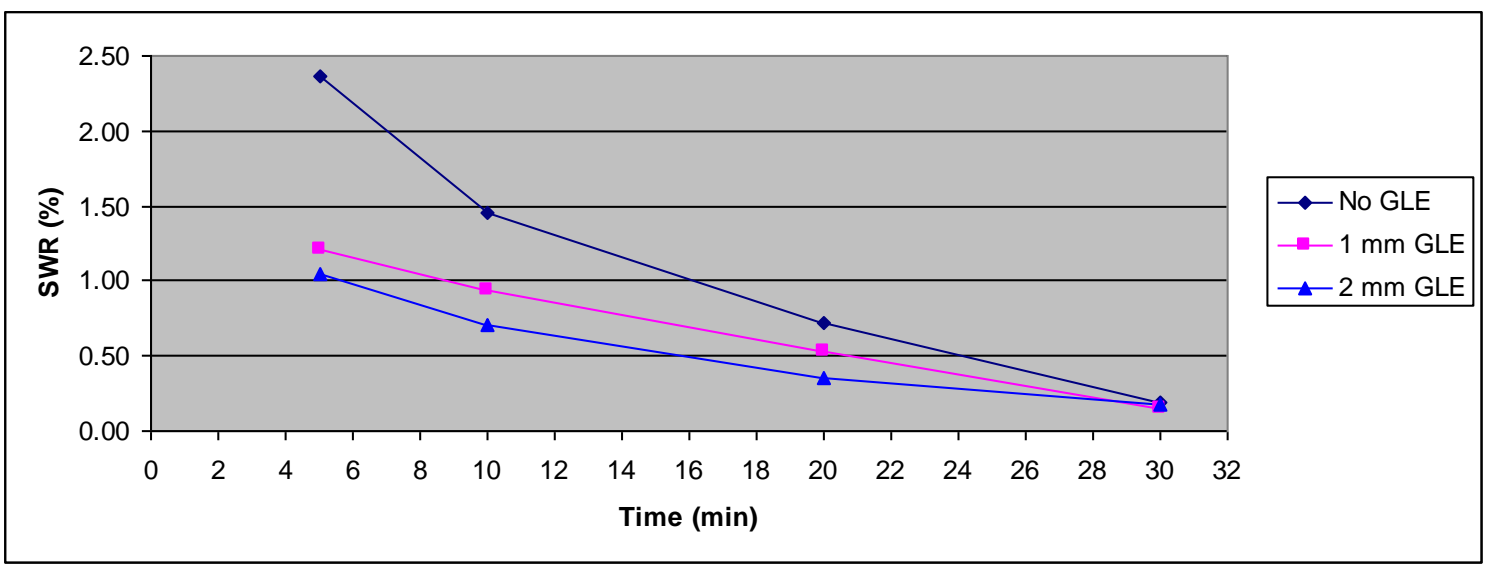

Figure 4. GLE and etching time correlation.

It can be observed from figure 4 that, at only 5 minutes into the etching time, the reflectivity of both samples with GLE are almost 1/2 of the one without the presence of GLE. With no GLE, around 15 minutes are needed to reach this level of reflectivity. This means that we can achieve the same reflectivity in almost $1 / 3$ the time that is needed without applying GLE. 


\subsection{Optimal \% IPA and GLE Combination}

We can see that the use of GLE would already allow us to obtain very low reflectivity without even using IPA as an active agent for texturing the silicon surface. However, it may also be observed that a combined use of both parameters, IPA and GLE, will result in obtaining even better performance than employing each of these parameters alone. Although the presence of the IPA is less critical to obtain low reflectance surfaces when GLE is used, it still may be seen that a combination of IPA and GLE results in the optimal outcome.

A performance study was done to determine the optimal IPA concentration in the presence of GLE at 2\% TMAH. The 2\% TMAH concentration had been found to be the optimal concentration in most of the experiments conducted previously and also supported by the literature [2].

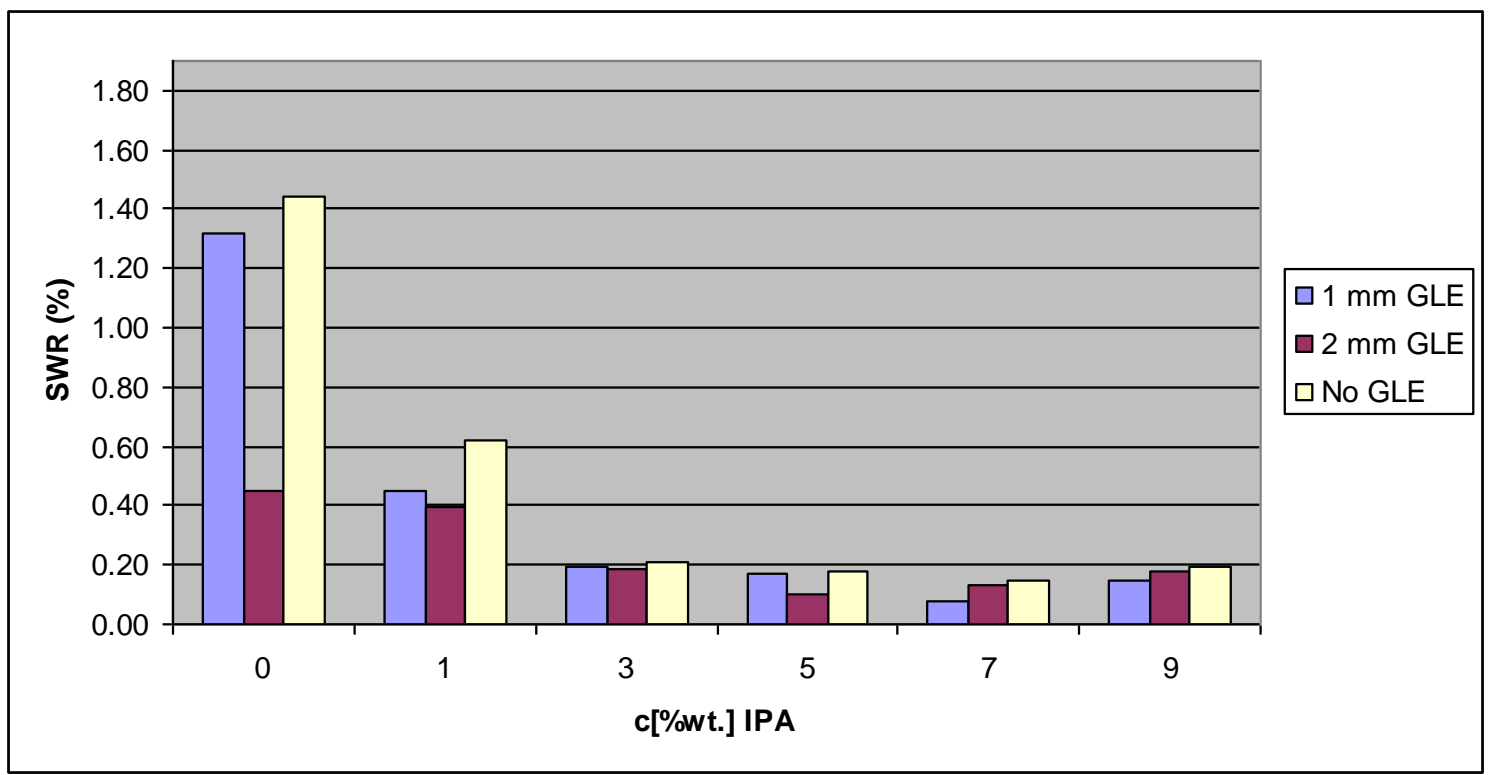

Figure 5. Optimal c[\%wt.] IPA and GLE combination.

In our experiments, the lowest reflectivity with no GLE present was found to be at 7\% IPA as illustrated in figure 5. The goal is to predict a possible optimal combination of IPA concentration and GLE that can achieve the same or better reflectivity as the 7\% IPA with no GLE. This combination must also allow the use of the lowest possible amount of IPA concentration leading to the reduction of the texturing cost. An experiment was conducted to confirm this prediction with the following parameter values: $2 \% \mathrm{TMAH}, 30 \mathrm{~min}, 90^{\circ} \mathrm{C}, 3.5 \%$ IPA. The experiment was conducted in the presence of 2-mm gap GLE.

The reflectivity of the textured silicon obtained under this condition is compared with others in figure 6. The obtained data confirmed our prediction that a combination of $3.5 \%$ IPA concentration and the presence of 2-mm gap GLE results in the same reflectivity as the optimal reflectivity with no GLE obtained at 7\% IPA. 


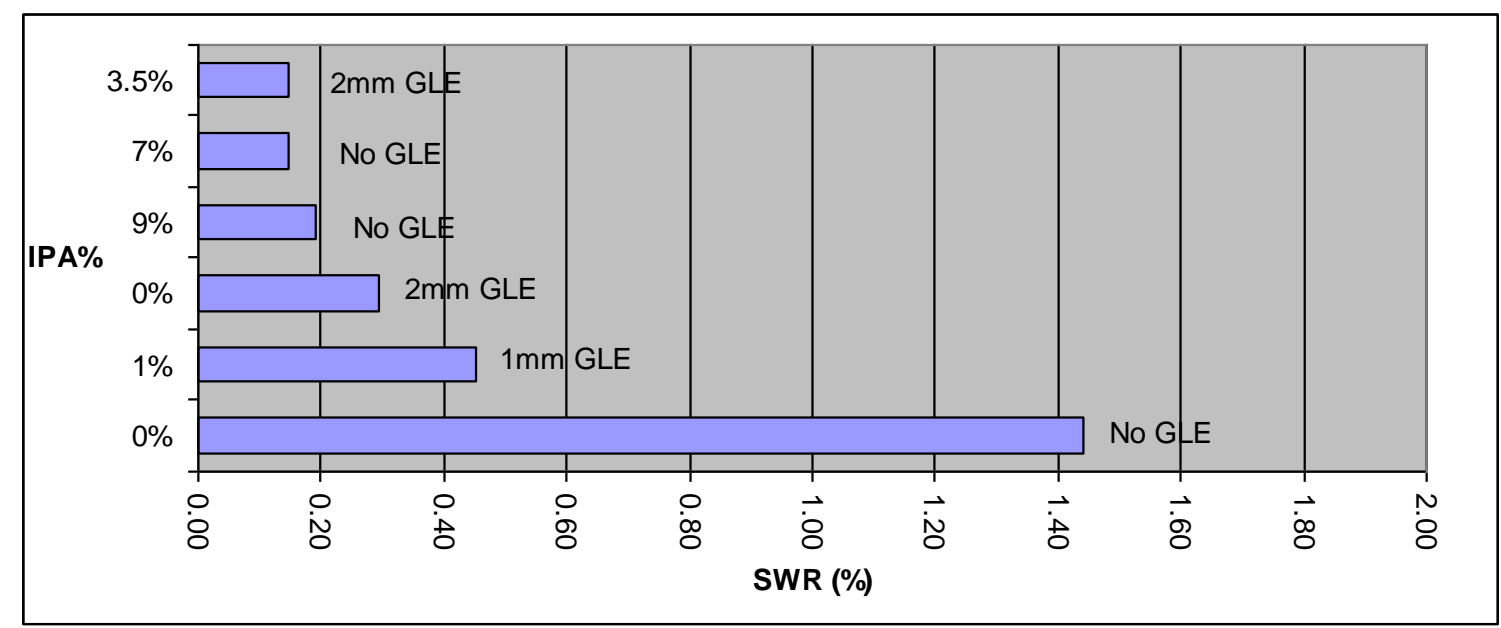

Figure 6. Optimal GLE-IPA\% combination resulting in $0.15 \%$ SWR.

\subsection{Correlation between Reflectivity and Etching Rate}

Figure 7 presents the behaviour of both reflectivity and the Etching Rate as the \%IPA increases with no GLE present.

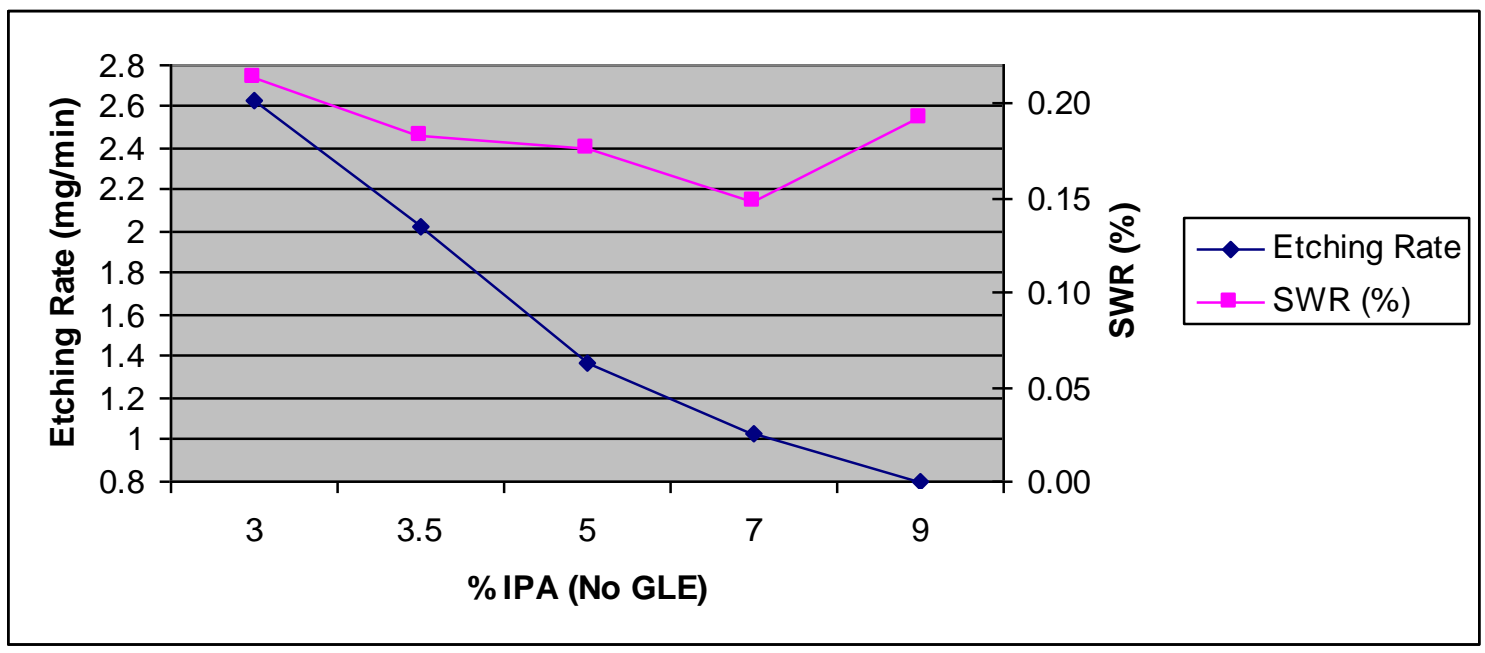

Figure 7. The behaviour of reflectivity and etching rate versus increasing \%IPA with no GLE present.

As expected, up to a certain point, both reflectivity and etching rate decrease as \%IPA increases. However, at around 7\% IPA, as the etching rate continues to drop, the reflectivity starts to increase. One possible explanation for this behaviour is that, beyond this optimal IPA concentration, the surface coverage will start to decrease resulting in higher reflectivity. This is also in agreement with our earlier conclusion that reflectivity is dependent on both uniformity and the surface coverage. We believe that, up to 7\% IPA, the uniformity and surface coverage are both improving. However, after that point, while the etching rate is still dropping contributing to the uniformity, the increasing IPA concentration starts to have a blocking effect on the etchant to have good surface contact. For the case where there is 2-mm gap GLE, this optimal IPA concentration is shifted to a lower value (around 5\%) as shown in figure 8 . From this point on, the reflectivity starts to increase as the etching rate still continues to drop. 


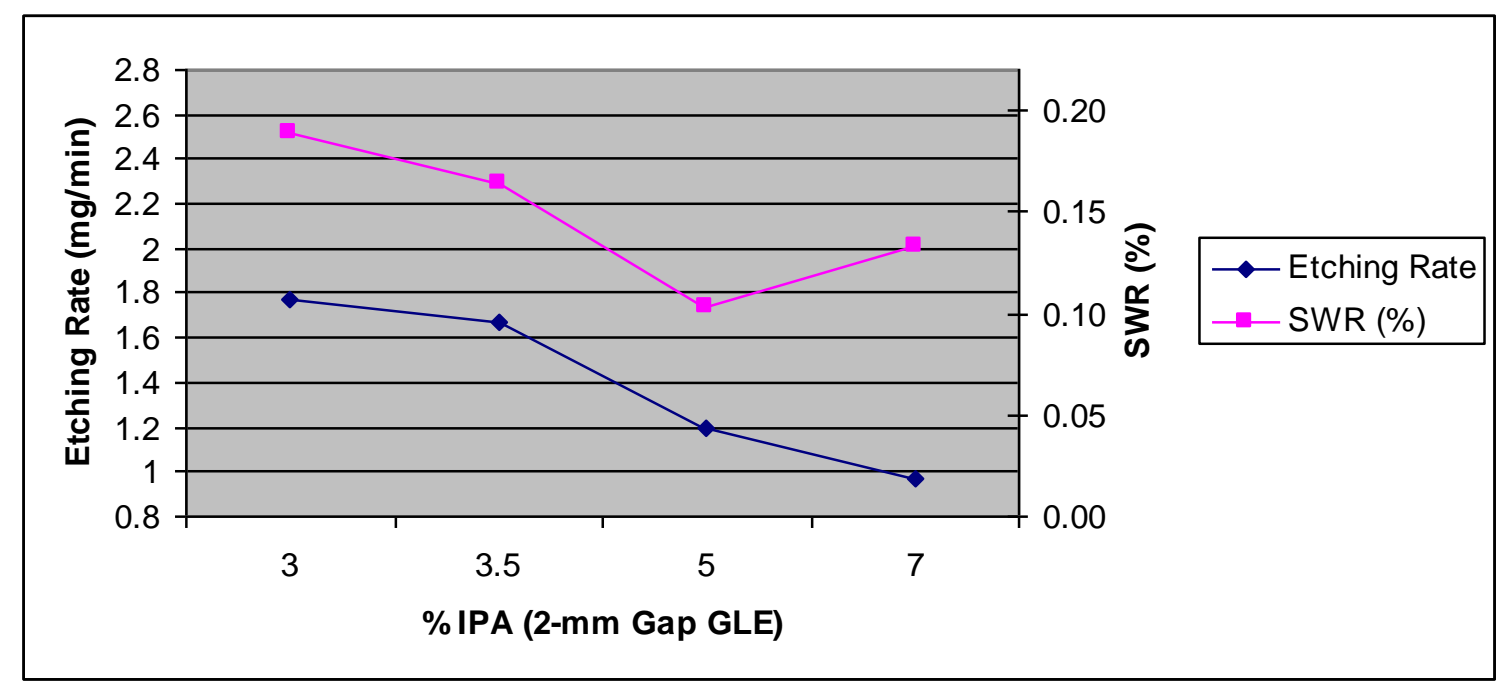

Figure 8. The behaviour of reflectivity and etching rate versus increasing \%IPA with $2 \mathrm{~mm}$ GLE present.

\subsection{Correlation between reflectance and morphology}

To evaluate any possible correlation between the measured reflectivity values of the samples and their morphology, an image analysis tool called ImageJ was used to calculate the surface coverage of pyramids and the standard deviation of the their sizes.

The samples listed in table 2 were all etched under the following parameter conditions: $2 \%$ TMAH, 30 min and $90^{\circ} \mathrm{C}$. The three samples having practically within the same particle size range were selected to evaluate any possible correlation between surface reflectivity and coverage. Table 2 clearly shows that as the surface coverage decreases, the reflectivity increases as expected.

Table 2. The correlation between reflectivity and surface coverage.

\begin{tabular}{|l|c|c|c|}
\hline & $\begin{array}{c}2 \mathrm{~mm} \text { GLE, } \\
3.5 \% \text { IPA }\end{array}$ & $\begin{array}{c}2 \mathrm{~mm} \text { GLE, } \\
0 \% \text { IPA }\end{array}$ & $\begin{array}{c}\text { No GLE, } \\
\text { 0\% IPA }\end{array}$ \\
\hline SWR $(\%)$ & 0.16 & 0.45 & 1.4 \\
\hline Surface Coverage $(\%)$ & 93.4 & 92.5 & 72.3 \\
\hline Mean Particle Size $\left(\mu^{2}\right)$ & 4 & 2.7 & 3 \\
\hline
\end{tabular}

However, as conditions (a) and (b) in table 3 illustrate, the surface coverage is not the only parameter that influences the measured reflectivity. In fact, a significant difference in average particle size results in a considerable difference in surface reflectivity even for the same surface coverage. It may also be noted that the sample with the lower surface reflectivity presents a more uniform surface as it may be confirmed by the values of standard deviation of the particle size in table 3 . This observation suggests that both surface coverage and particle uniformity influence the reflectivity of the surface. The above point can also be confirmed based on the conditions (c) and (d) in table 3 . The data show that for slightly lower coverage, smaller particle size and smaller standard deviation result in a lower reflectivity. 
Table 3. Influence of particle size and uniformity on reflectivity.

\begin{tabular}{|c|c|c|c|c|}
\hline Experiment Condition & $a^{*}$ & $b^{*}$ & $c^{*}$ & $\mathrm{~d}^{*}$ \\
\hline SWR (\%) & 0.15 & 0.45 & 0.19 & 0.45 \\
\hline Surface Coverage $(\%)$ & 87.1 & 90.9 & 91.0 & 92.5 \\
\hline Standard Deviation $\left(\mu^{2}\right)$ & 0.53 & 13.6 & 1.38 & 3.92 \\
\hline Mean Particle Size $\left(\mu^{2}\right)$ & 0.52 & 13.21 & 0.52 & 2.7 \\
\hline
\end{tabular}

Figures $9 \mathrm{a}$ and $9 \mathrm{~b}$ illustrate the surface morphology of the two samples listed in table 3 as (c) and (d), respectively. As mentioned before, the coverage is slightly higher in the sample presented in figure $9 \mathrm{~b}$. However, a more uniformity and smaller particle size (figure 9a) have resulted in lower reflectivity. One should note that the two SEM pictures have different magnifications and do not have the same scale. The ImageJ tool takes the difference of scale into account when calculating and comparing the surface coverage and particle size uniformity.

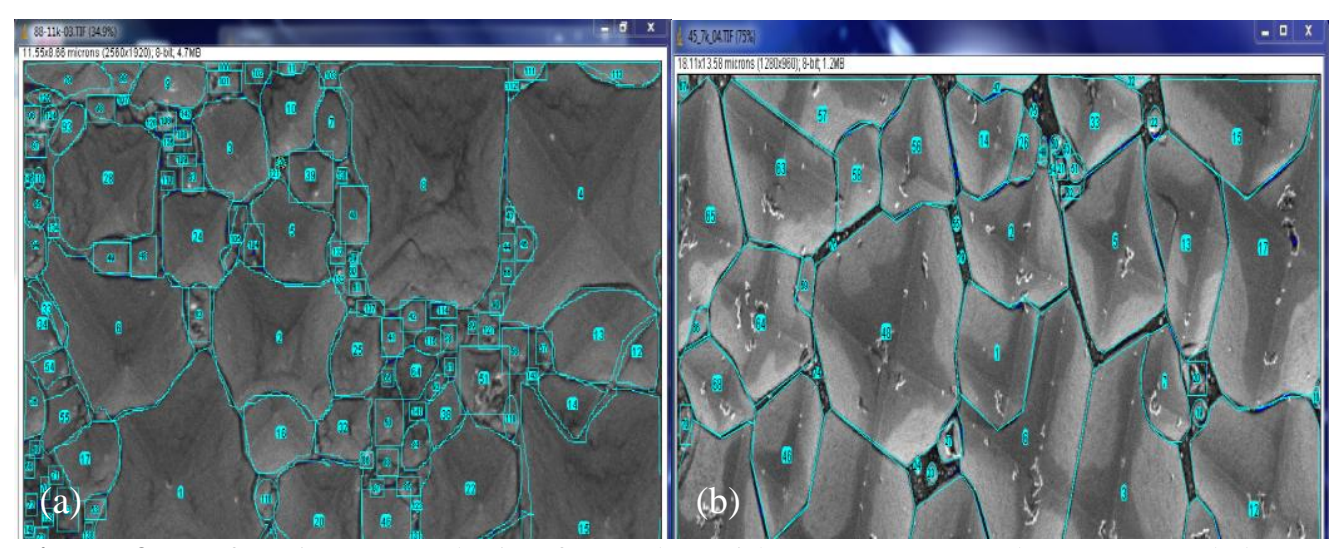

Figure 9. Surface image analysis of samples with (a) no GLE and 9\% IPA and (b) 2mm GLE and $0 \%$ IPA.

\subsection{Effect of etching time on morphology}

As it was shown earlier in section 3.4, with shorter etching time we can achieve the same reflectivity using GLE. This phenomenon can also be explained by looking at the morphology of the surface of the samples with and without the presence of GLE. The SEM photos in figure 10 are taken from samples under the following etching parameters condition: 1\% IPA, 2\% TMAH and $90^{\circ} \mathrm{C}$ temperature. This figure compares the surface coverage and uniformity of two samples at 10 minutes into the etching time, one with (figure 10a) and one without (figure 10b) the presence of GLE. One very clear observation is the surface damages shown in the figure $10 \mathrm{~b}$. We speculate that these damages are due to hydrogen bubbles sticking to the surface of silicon in the absence of GLE. Those damaged areas are not observed when GLE is present (figure 10a). 


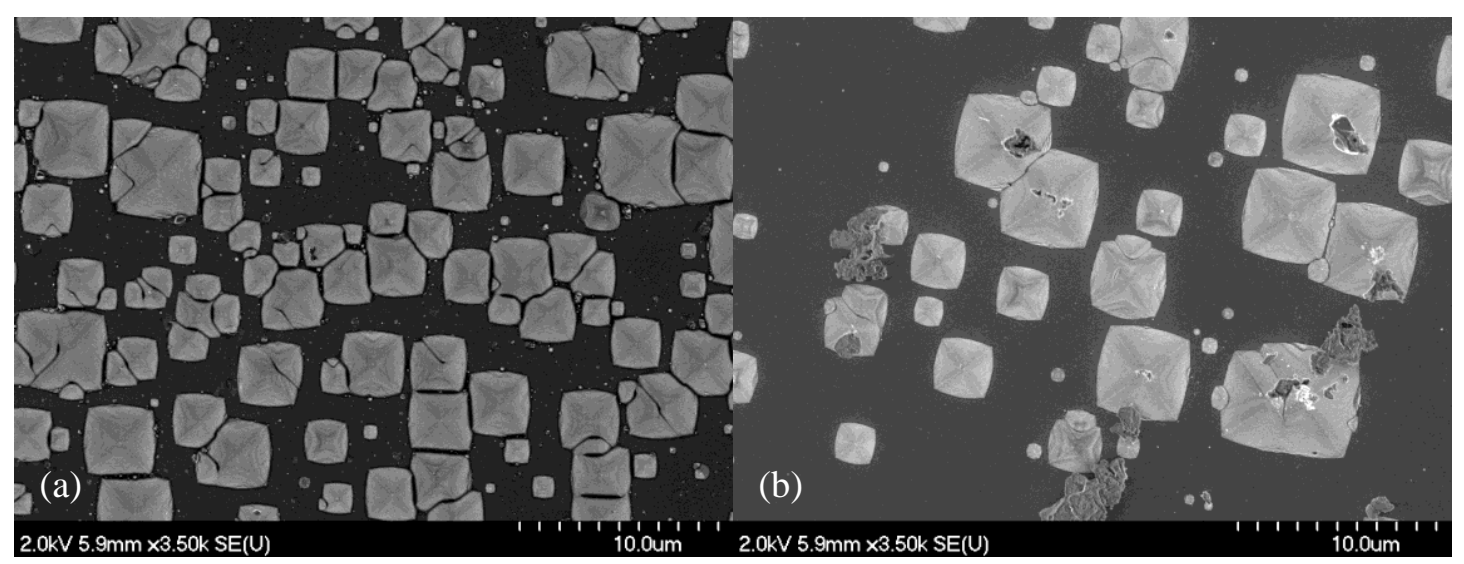

Figure 10. SEM photos of textured silicon at 10 minutes into the etching time with 1\% IPA and $2 \%$ TMAH, (a) presence of 2 mm GLE (b) absence of GLE

The result of performing reflectivity measurements of both samples as well as image analysis of their SEM photos are summarized in table 4 in terms of reflectivity, standard deviation, surface coverage and the average particle size.

Table 4. Reflectivity, standard deviation, and surface coverage at 10 minutes etching time.

\begin{tabular}{|l|c|c|c|c|}
\hline & $\begin{array}{c}\text { SWR } \\
(\%)\end{array}$ & $\begin{array}{c}\text { Surface Coverage } \\
(\%)\end{array}$ & $\begin{array}{c}\text { StDev } \\
\left(\mu^{2}\right)\end{array}$ & $\begin{array}{c}\text { Average Particle } \\
\text { Size }\left(\mu^{2}\right)\end{array}$ \\
\hline 2mm GLE & 13.9 & $51.4 \%$ & 3.53 & 2.42 \\
\hline No GLE & 29.7 & $28.2 \%$ & 7.06 & 4.82 \\
\hline
\end{tabular}

As it may be seen, the use of GLE resulted in a significantly lower reflectivity after 10 minutes into the experiment compared to the sample with no GLE. The analysis of the morphology confirms the previously mentioned observation that the sample with lower reflectivity presents also higher surface coverage, a lower mean and higher uniformity of the particle size.

\section{Conclusions}

In this study, etching experiments were performed on n-type (100) silicon wafers with and without the presence of Gas Lift Effect (GLE). In the presence of GLE, the need for the use of a surface active agent, such as IPA, to help minimize the surface reflectance becomes much less critical. In fact, GLE can achieve the same reflectivity with 50\% the amount of IPA, compared to the case where no GLE is present. It can also reduce the processing time by more than $60 \%$ to achieve the same reflectivity. Although the presence of IPA is less critical to obtain low reflectance surfaces when GLE is used, it was concluded that a combination of IPA and GLE achieved the lowest reflectivity. Accordingly, a specular solar-weighted reflectance (SWR) of $0.15 \%$ was achieved with a combination of $3.5 \%$ IPA and $2 \mathrm{~mm}$ GLE. The morphology of the surface was studied by performing image analysis of the SEM photos of the textured surface. The correlation between parameters such as surface coverage, uniformity and particle size with the reflectivity was studied and it was concluded that reflectivity is highly influenced by those parameters. A significant difference in average particle size results in a considerable change in surface reflectivity even at the same surface coverage. Lower reflectivity is a result of a combination of lower mean particle size as well as higher surface coverage and uniformity of the particles. The use of this new approach in surface texturing reduces the IPA and time required to reach the same reflectivity, and therefore, reducing the cost and increasing the efficiency of the texturing process. 


\section{Acknowledgments}

We are grateful to Dr. Laszlo Kalman for allowing us to use the spectrophotometer equipment in his lab at the physics department of Concordia University and his useful discussions and suggestions.

\section{References}

[1] Green M. A., Solar Cells: Operating Principles, Technology and System Application, volume 1, University of New Wales, Kensington, 1982.

[2] Papet P, Nichiporuk O, Fave A, Kaminski A, Bazer-Bachi B, Lemiti M 2006 TMAH texturisation and etching of interdigitated back-contact solar cells Materials Science 24 10431049

[3] Merlos A, Acero M, Bao M H, Bausells J and Esteve J 1993 TMAH/IPA anisotropic etching characteristics Sensors and Actuators A: Physical 37-38 737-743

[4] Zubel I, Barycka I, Kotowska K, Kramkowaska M, 2001 Silicon anisotropic etching in alkaline solutions IV, The effect of organic and inorganic agents on silicon anisotropic etching process Sensors and actuator A 87 163-171

[5] Zubel I, Kramkowska M 2001 The effect of isopropyl alcohol on etching rate and roughness of (100) Si surface etched in KOH and TMAH solutions Sensors and actuator A 93 138-147 [6] Ou Weiying, Zhang Yao, Li Hailing, Zhao Lei, Zhou Chunlan, Diao Hongwei , Liu Min, Lu Weiming, Zhang Jun and Wang Wenjing 2010 Texturization of mono-crtystalline silicon solar cells in TMAH without the addition of surfactant Journal of Semiconductors 31106002 [7] Soonwoo kwon, Jongheop Yi, Sewang Yoon, Joon Sung Lee, Donghwan Kim 2009 Effects of textured morphology on the short circuit current of single crystalline silicon solar cells:

Evaluation of alkaline wet-texture process Current Applied Physics 9 1310-1314

[8] Chii-Rong Yang, Chen-Hao Yang and Po-Ying Chen 2005 Study on anisotropic silicon etching characteristics in various surfactant-added tetramethyl ammonium hydroxide water solutions Journal of Microelectronics and Microengineering 15 2028-2037

[9] Chu A K, Wang J S, Tsai Z Y, Lee C K 2009 A simple and cost-effective approach for fabricating pyramids on crystalline silicon wafers Solar Energy Materials \& Solar Cells 93 12761280

[10] Vogt H, Kreysa G 2008 Electrochemical Reactors Ullmann's Encyclopedia of Industrial Chemistry

[11] Grotheer M P 2000 Electrochemical Processing, Inorganic Kirk-Othmer Encyclopedia of Chemical Technology 9 618-652

[12] Chii-Rong Yang, Po-Ying Chen, Cheng-Hao Yang, Yuang-Cherng Chiou, Rong-Tsong Lee 2005 Effects of various ion-typed surfactants on silicon anisotropic etching properties in $\mathrm{KOH}$ and TMAH solutions Sensors and Actuators A 119 271-281 